\title{
$H$-COBORDISMS WITH FOLIATED CONTROL
}

\author{
F. T. FARRELL AND L. E. JONES
}

\begin{abstract}
We announce a foliated version of Ferry's metric $h$-cobordism theorem [13]. Let $M$ be a compact Riemannian manifold and $\mathcal{F}_{\text {a }}$ smooth foliation of $M$ such that the sectional curvatures of the leaves of $\mathcal{F}$ are zero. There are numbers $\alpha>0$ (depending only on $\operatorname{dim}(M))$ and $\delta>0$ (depending on $M$ and $\mathcal{F}$ ) so that if $W$ is an $h$-cobordism over $M$ having $\delta$ control in the directions perpendicular to $\mathcal{F}$ and having $\alpha \cdot i(\mathcal{F})$ control in the directions tangent to $\mathcal{F}$, then $W$ is a product cobordism. Here $i(\mathcal{F})$ denotes the greatest lower bounds for the injectivity radii of all the leaves of $\mathcal{F}$.
\end{abstract}

Statement of theorems. $M$ will denote a smooth compact Riemannian manifold and $\mathcal{F}$ will denote a smooth foliation of $M$. Note that each leaf $L$ of $\mathcal{F}$ inherits a Riemannian structure from $M$, and therefore has a well-defined radius of injectivity $i(L)(\mathrm{cf}$. [5]). We define $i(\mathcal{F})$ to be the greatest lower bound for all the $i(L)$.

$W$ will denote an $h$-cobordism with $\partial_{-} W=M . W$ comes equipped with homotopy retractions $h^{-}: W \times[0,1] \rightarrow W$ and $h^{+}: W \times[0,1] \rightarrow W$, satisfying: $h^{-}(W \times 1) \subset \partial_{-} W, h^{-}(x, t)=x$ for all $x \in \partial_{-} W$ and $t \in[0,1] ; h^{+}(W \times 1) \subset$ $\partial_{+} W, h^{+}(x, t)=x$ for all $x \in \partial_{+} W$ and $t \in[0,1]$.

To any continuous path $p:[0,1] \rightarrow M$ we can associate two numbers $L_{1}(p)$, $L_{2}(p)$ as follows. $L_{2}(p)$ is the greatest lower bound of all numbers $\lambda>0$ that satisfy: there is a continuous path $q:[0,1] \rightarrow L$ into some leaf $L$ of $\mathcal{F}$ such that $d(q(t), p(t)) \leq \lambda$ for all $t \in[0,1]$ (here $d(,$,$) denotes the metric on M$ induced by the Riemannian structure). Define $L_{1}(p)$ to be the greatest lower bound of all numbers $\lambda>0$ that satisfy: there is a continuous path $q:[0,1] \rightarrow L$ into a leaf of $\mathcal{F}$ such that $d(q(t), p(t)) \leq 2 L_{2}(p)$ for all $t \in[0,1]$; moreover the diameter of $q([0,1])$ in $L$ is less than or equal to $\lambda$.

Using the $L_{1}()$ and $L_{2}()$ we can now define the diameter of the $h$ cobordism $W$ in the direction parallel to $\mathcal{F}$-denoted by $D_{1}(W)$-and in the direction perpendicular to $\bar{F}$-denoted by $D_{2}(W)$. For each $y \in W$ let $p_{y}^{-}:[0,1] \rightarrow M$ denote the composition

$$
[0,1]=(y) \times[0,1] \subset W \times[0,1] \stackrel{h^{-}}{\rightarrow} W=W \times 1 \stackrel{h^{-}}{\rightarrow} \partial_{-} W=M ;
$$

and let $p_{y}^{+}:[0,1] \rightarrow M$ denote the composition

$$
[0,1]=(y) \times[0,1] \subset W \times[0,1] \stackrel{h^{+}}{\rightarrow} W=W \times 1 \stackrel{h^{-}}{\rightarrow} \partial_{-} W=M .
$$

Received by the editors January 12, 1986 .

1980 Mathematics Subject Classification (1985 Revision). Primary 18F25, 57Q10, 57R80.

Both authors were supported in part by the NSF. 
Define $D_{i}(W)$ to be the least upper bound of all the numbers $\left\{L_{i}\left(p_{y}^{-}\right), L_{i}\left(p_{y}^{+}\right)\right.$: $y \in W\}$.

The following theorem is a generalization of Ferry's metric $h$-cobordism theorem [13]. (Note that the following theorem becomes Ferry's theorem if the foliation has single points for leaves.)

THEOREM A. Suppose that $\operatorname{dim}(M) \geq 5$, and each leaf of $₹$ has zero sectional curvature. Then there is a number $\alpha$ such that the following are satisfied:

(a) $\alpha$ depends only on $\operatorname{dim}(M) ; 0<\alpha<1$.

(b) Given any $\beta>0$ with $\beta<\alpha \cdot i(\mathcal{F})$, there is $\delta>0$ so that if $D_{1}(W) \leq \beta$ and $D_{2}(W) \leq \delta$ then $W$ is a product cobordism.

We have also been able to generalize the local contractibility results of Edwards and Kirby [7], and Chernavskii [2], to a foliated context. Let $\mathcal{H}(M)$ denote the space of homeomorphisms of $M$. For any numbers $\alpha>0$ and $\delta>0$ there is a subspace $\sharp^{\alpha, \delta}(M) \subset \mathcal{H}(M)$ defined as follows. A homeomorphism $f: M \rightarrow M$ is in $\nvdash^{\alpha, \delta}(M)$ if for every $y \in M$ there is a path $q:[0,1] \rightarrow M$ with $q(0)=y, q(1)=f(y)$ and such that $L_{1}(q) \leq \alpha, L_{2}(q) \leq \delta$.

THEOREM B. Suppose that the leaves of $\mathcal{F}$ have zero sectional curvatures. There are numbers $\alpha, \alpha^{\prime}$ which satisfy the following:

(a) $\alpha, \alpha^{\prime}$ depend only on $\operatorname{dim}(M) ; 0<\alpha, \alpha^{\prime}<1$.

(b) Given any $\beta, \varepsilon>0$ with $\beta<\alpha \cdot i(\mathcal{F})$, there are $\beta^{\prime}, \delta>0$ with $\beta^{\prime}=\alpha^{\prime} \cdot \beta$ and a homotopy $h: \mathcal{H}^{\beta^{\prime}, \delta}(M) \times[0,1] \subset \mathfrak{H}^{\beta, \varepsilon}(M)$ satisfying: $h\left(1_{M}, t\right)=1_{M}$ for all $t \in[0,1] ; h\left(\nvdash^{\beta^{\prime}, \delta}(M) \times 1\right)=1_{M}$.

Note that if $\mathcal{F}$ has single points for leaves, then Theorem B is the local contractibility theorem of Edwards and Kirby [7].

Related results. Theorem A has actually been proved in $[\mathbf{1 2}]$ in much greater generality than has been described above. We give here only a rough description of this more general theorem, by describing in (1)-(4) below the more general properties of $M, \mathcal{F}$, and $W$ that still insure the truth of Theorem A.

(1) $M$ is a smoothly stratified space, having Riemannian manifolds for strata. $M$ need not be compact.

(2) $\mathcal{F}$ consists of a collection of smooth foliations $\left\{\mathcal{F}_{i}\right\}$, one for each of the strata $\left\{M_{i}\right\}$ of $M$. Moreover the $\left\{\mathcal{F}_{i}\right\}$ are interrelated so as to form a "stratified foliation" for $M$.

(3) $p: E \rightarrow M$ is a continuous map from a manifold $E$ satisfying: each restriction $p: p^{-1}\left(M_{i}\right) \rightarrow M_{i}$ is a fiber bundle with fiber equal to a compact closed manifold $F_{i} ; \operatorname{Wh}\left(\pi_{1}\left(F_{i}\right) \oplus A\right)=0$, for any $i$ and any finitely generated torsion free abelian group $A$.

(4) $W$ is an $h$-cobordism with $\partial_{-} W=E$, which is a product cobordism over the complement of a compact subset of $E$.

In this more general context the diameters $D_{1}(W)$ and $D_{2}(W)$ are still measured in $M$ by projecting under $p: E \rightarrow M$ all the relevant homotopy retraction trails in $E$. This formulation of Theorem A generalizes the fibered 
version of Ferry's metric $h$-cobordism theorem which has been proved by Chapman [3] and Quinn [16].

The above generalization of Theorem $\mathrm{A}$ is important because it allows us, in many useful special cases, to deal with foliated control problems for which $D_{1}(W)$ is much larger than $i(\mathcal{F})$ but $D_{2}(W)$ is very small. The simplest such example occurs when $\mathcal{F}$ is a one-dimensional foliation of the compact Riemannian manifold $M$, satisfying:

(5) For any given $k>0$ there are only a finite number of leaves in $₹$ having length less than or equal to $k$.

Note that by collapsing a finite number of closed leaves $\left\{L_{i}\right\}$ of $₹$ to points $\left\{\left[L_{i}\right]\right\}$, we obtain a stratified quotient space $M^{\prime}$ equipped with a stratified foliation $F^{\prime}$ : the strata of $M^{\prime}$ consist of $M-\bigcup_{i} L_{i}$, and the finite set $\bigcup_{i}\left[L_{i}\right]$; $F^{\prime}$ equals $\mathcal{F}$ on $M-\bigcup_{i} L_{i}$, and has single points for leaves in $\bigcup_{i}\left[L_{i}\right]$. If enough of the shorter closed leaves of $\mp$ have been collapsed to points, then $D_{1}(W)$ as measured in $M^{\prime}$-will be much less than $i\left(\mathcal{F}^{\prime}\right)$, and $D_{2}(W)$-as measured in $M^{\prime}$ - will still be small. So the stratified version of Theorem $\mathrm{A}$ may be applied to $W, M^{\prime}, \mathcal{F}^{\prime}$, to complete the proof of the following corollary.

COROllary C. Let $M, \mp$ be as in (5) above. For any $\alpha>0$ there exists $\varepsilon>0$ so that if $D_{1}(W) \leq \alpha$ and $D_{2}(W) \leq \varepsilon$ are both true for an $h$-cobordism $W$ over $M$, then $W$ is a product cobordism.

In sequels to $[\mathbf{1 0}, \mathbf{1 1}$, and $\mathbf{1 2}]$, we shall extend other types of metric control theorems to the foliated context. For example, if we drop the hypothesis "Wh $\left(\pi_{1}\left(F_{i}\right) \oplus A\right)=0$ " from (3) above, then we can obtain a foliated version of a result due to Quinn [17] and Chapman [4], which locates the Whitehead torsion of an $h$-cobordism (over the total space of a bundle $E \rightarrow M$ with foliated control in $M$ ) in the homology of $M$ with coefficients in the Whitehead group, projective class group, and lower $K$-groups of the fundamental group of the fiber.

Proofs of theorems. In principle the proofs of all metric control theorems follow the pattern set down by Edwards and Kirby in their proof of the local contractibility of the space of homeomorphisms of a manifold [7]. We recall that the two steps in this pattern of proof are as follows.

Step 1. Choose a handle body for $M$, and choose the upper bounds for the metric control to be much less than the diameters of the handles of $M$.

Step 2. Proceed inductively over the dimension of the handles, using the torus trick to obtain the desired result in each handle.

Our proofs for Theorems A, B follow the above pattern of proof, with some exceptions which we shall describe now. Not just any handle body will do in Step 1, because we only allow the upper bounds for metric control to be chosen arbitrarily small in the directions perpendicular to the foliation $\mathcal{F}$. For this reason we must choose the handle body for $M$ so that each handle has diameter greater than $\gamma \cdot i(\mathcal{F})$ in the directions tangent to $\mathcal{F}$, where $\gamma$ is a positive number depending only on $\operatorname{dim}(M)$. Then choose $\alpha$ of Theorems $\mathrm{A}$, $\mathrm{B}$ to be much smaller than $\gamma$; and choose the upper bounds $\delta$, for the metric control in directions perpendicular to $\mathcal{F}$, to be much smaller than the diameter 
of the handles in these same directions. In Step 2, instead of applying the torus trick, we simply scale down the metric in each handle in the direction of $\mathcal{F}$, and scale up the metric in the direction perpendicular to $\mathcal{F}$. In this way we obtain the standard metric on each handle, with respect to which the control data is no longer foliated control, but rather just ordinary control. So our version of Step 2 above can be completed by just applying the relevant control theorem (for Theorem A we apply Ferry's theorem, for Theorem B we apply the theorem of Edwards and Kirby).

\section{REFERENCES}

1. D. V. Anosov, Geodesic flows on closed Riemannian manifolds with negative curvature, Proc. Steklov Inst. Math. no. 90, Amer. Math. Soc., Providence, R. I., 1969.

2. A. V. Chernavskii, Local contractibiility of the homeomorphism group of a manifold, Soviet Math. Dokl. 9 (1968), 1171-1174.

3. T. A. Chapman, Homotopy conditions which detect simple homotopy equivalences, Pacific J. Math. 80 (1979), 13-46.

4. Controlled simple homotopy theory and applications, Lecture Notes in Math., vol. 1009, Springer-Verlag, Berlin, 1983.

5. J. Cheeger and D. Ebin, Comparison theorems in Riemannian geometry, NorthHolland Publishing Company, Amsterdam, 1975.

6. E. H. Connoll and J. Hollingsworth, Geometric groups and Whitehead torsion, Trans. Amer. Math. Soc. 140 (1969), 161-181.

7. R. D. Edwards and R. C. Kirby, Deformations of spaces of imbeddings, Ann. of Math. (2) 93 (1971), 63-88.

8. F. T. Farrell and L. E. Jones, Markov cell structures, Bull. Amer. Math. Soc. 83 (1977), 739-740.

9. , Markov cell structures for expanding maps in dimension two, Trans. Amer. Math. Soc. 255 (1979), 315-327.

10. , $K$-theory and dynamics. I (submitted for publication).

11. _ـ K-theory and dynamics. II (submitted for publication).

12. The Whitehead group of cocompact discrete subgroups of connected Lie groups (in preparation).

13. S. Ferry, The homomorphism group of a compact Hilbert cube manifold is an $A N R$, Ann. of Math. (2) 106 (1977), 101-119.

14. A. E. Hatcher, Concordance spaces, higher simple homotopy theory, and applications, Proc. Sympos. Pure Math. 32 (1978), 3-21.

15. J. Milnor, Morse Theory, Ann. of Math. Studies, No. 51, Princeton Univ. Press, Princeton, N.J., 1963.

16. F. Quinn, Ends of maps. I, Ann. of Math. 110 (1979), 275-331.

17. __, Ends of maps. II, Invent. Math. 68 (1982), 353-424.

Department of Mathematics, Columbia University, New York, NeW YORK 10027

Department of MATHEMATICS, State University OF NEW YORK AT STONY BROOK, STONY BROOK, NEW YORK 11794 